\title{
Clinical Equipoise
}

National Cancer Institute

\section{Source}

National Cancer Institute. Clinical Equipoise. NCI Thesaurus. Code C142428.

A state of uncertainty regarding the therapeutic advantages of one treatment over another. 